\title{
Quality and standardization in blood component preparation with an automated blood processing technique
}

\author{
D. J. van Rhenen, $* \dagger$ J. Vermeij, $*$ J. de Voogt,* J. C. Bernes* and J. M. Payrat $\$ *$ Bloodbank Rotterdam, \\ $\dagger$ Department of Haematology, University Hospital Rotterdam, the Netherlands, and $\ddagger$ Baxter R \& D Europe SC, Nivelles, Belgium
}

Received 26 January 1998; accepted for publication 10 July 1998

SUMMARY. The use of automated blood processors in combination with bottom and top blood containers has been found to improve the standardization and quality of blood components. A study was performed to validate a new type of processor (Optipress ${ }^{\circledR}$ II) and compare its performance with a first generation processor (Optipress $®$ I).

Primary separation on the Optipress II was investigated on $570 \mathrm{~mL}( \pm 10 \%)$ of anticoagulated blood in a nonpaired study. In addition, the quality of the products in routine production was compared between the results of the Optipress I and Optipress II. The whole blood units were kept overnight at room temperature $\left(20 \pm 2{ }^{\circ} \mathrm{C}\right)$. Separation was performed under conditions to obtain $55 \mathrm{~mL}$ buffy coats with a $50 \%$ haematocrit (ht). Platelet concentrate preparation was investigated in a paired study and compared to the routine manual method using PAS II additive solution. Parameters studied were volume, red cell, white cell and platelet counts, ht, haemoglobin (hb, total and free).

Primary separation was more efficient in the Optipress II because the platelet count was lower in the erythrocyte concentrates $(P<0.0001)$, platelets were lower in plasma $(P<0.0001)$ and platelet counts were higher in buffy coats $(P<0.0001)$. Buffy coat volume showed less variation (Optipress II $\mathrm{VC}=4 \%$, Optipress I VC= $7 \cdot 4 \%$ ). Secondary separation did not show differences between the Optipress II and manual method but was advantageous because of the automatic termination of the procedure.

Further improvement of standardization in blood component preparation is possible with an automated blood processor, leading to improvement of the quality of blood products for patient care.

Key words: automated blood processors, blood component preparation, standardization.
Blood component preparation has become a routine procedure worldwide (Heaton et al., 1997). Basically whole blood is collected in anticoagulant solutions and centrifuged to produce components adapted to the user's need. The aim can be to optimize buffy coat separation, to maximize plasma harvest or to maximize red cell recovery with an acceptable plasma harvest. Generally the recovered red cells are suspended in additive solution (AS) (Lovric et al., 1977; Högman et al., 1978) to optimize storage conditions and maximize plasma harvest. A similar approach of AS addition is increasingly applied to platelet concentrates prepared from buffy coats (Gulliksson et al., 1995; van Rhenen et al., 1995).

An ever increasing quality of blood components can thus be offered to the hospitals, produced in an efficient way (Molijn et al., 1994). The basic principle of this development is standardization. The whole blood volume is collected within specified borders $( \pm 10 \%)$ and the

Correspondence: D. J. van Rhenen, MD PhD, Bloodbank Rotterdam, PO Box 23370, 3001 KJ Rotterdam, the Netherlands.

(C) 1998 Blackwell Science Ltd component preparation is performed according to GMP rules and standardized within specified limits regarding volume, haematocrit, white cell and platelet count.

The use of bottom and top containers in combination with automated component separation devices has been found to improve further standardization and the quality of blood components (Heaton et al., 1997).

In this study we compared the performance of one of the first component separation devices available (Optipress ${ }^{\circledR}$ I) with a recently developed automated device (Optipress ${ }^{\circledR}$ II) to investigate whether standardization and quality of blood components could be improved further.

\section{MATERIALS AND METHODS}

\section{Blood packs}

Optipac ${ }^{\circ}$ (Baxter Inc., La Châtre, France) was used for collecting $500 \pm 50 \mathrm{~mL}$ blood from volunteer donors. 
The blood pack consists of a primary 'bottom and top' bag filled with $70 \mathrm{~mL}$ CPD anticoagulant solution, a secondary conventional bag filled with $110 \mathrm{~mL}$ SAG-M solution and an empty transfer bag. Packs are made of PL 2209 plastic material.

\section{Blood processors}

A machine called Optipress II was developed for automated blood processing by Baxter R and D, Nivelles, Belgium. This device incorporates the functions available on its predecessor (Optipress I) but is enhanced by several modifications. For instance the machinery is not, as is the Optipress I, dependent on compressed air. Furthermore, the detection of the interface between cells and plasma has been improved and automated sealing can be performed.

\section{Study design}

The study was structured in four phases:

1 equipment performance validation for the primary and the secondary separation;

2 determination of the maximum press plate force setting;

3 Optipress II efficiency study;

4 comparison with Optipress I during routine production.

\section{Primary separation study}

Thirty-one blood units of $500 \mathrm{~mL} \pm 10 \%$ were collected from volunteer donors in $70 \mathrm{~mL}$ CPD anticoagulant solution using a 'bottom and top' system (Optipac). Selection of donors as well as blood donation procedures were based on local standard practices. All blood units were actively cooled to $20 \pm 2{ }^{\circ} \mathrm{C}$ and kept overnight at room temperature $\left(20 \pm 2{ }^{\circ} \mathrm{C}\right)$ before they were centrifuged at high speed $(4657 \mathrm{~g})$ for $10 \mathrm{~min}$.

The Optipress II was equipped with a 500 Opti $30 \mathrm{mid}$ backplate for the primary separation of $570 \mathrm{~mL}$ of anticoagulated blood using the following parameters: buffy coat volume: $55 \mathrm{~mL}$, buffy coat level: $6 \cdot 7$, pressplate force: $25 \mathrm{~kg}$. The same Optipress II was equipped with a flat backplate for the secondary separation of platelets from pooled buffy coats using the following parameters: time: 5 min, sensitivity: 40, red cell level (RCL): 40, pressplate force: $8 \mathrm{~kg}$.

The results used for comparison in the routine production study were those obtained in routine quality testing of the blood components produced on Optipress I during quarters 3 and 4 in 1995 and produced on Optipress II during quarters 3 and 4 in 1997.
Primary separation was performed under conditions defined to obtain $55 \mathrm{~mL}$ buffy coats with a $50 \%$ haematocrit.

Sampling was performed to obtain the following data: 1 whole blood donation: volume, red blood cell/white blood cell/platelet counts, haematocrit, total haemoglobin and free haemoglobin;

2 red cell concentrate: volume, total haemoglobin, red blood cell/white blood cell/platelet counts and haematocrit. Free haemoglobin was assessed at day $1(n=31)$, day $14(n=31)$ and day 35 of storage $(n=10)$;

3 plasma product: volume, total haemoglobin concentration, white cells and platelet counts;

4 buffy coat product: volume, red blood cell/white blood cell/platelet counts, total haemoglobin and haematocrit.

Automatic cell counting was performed for haemoglobin, red blood cell, white blood cell and platelets with a Cell Dyn 3500 cell counter (Abbott, Chicago, USA).

Leukocyte counting in plasma was performed in the Nageotte counting chamber technique.

\section{Secondary separation study}

This study was designed as a paired study comparing 12 separation processes on Optipress II vs. 12 manual separation processes. The Optipress II was equipped with a flat backplate for the separation of platelets from pooled buffy coats using the following parameters: time: $5 \mathrm{~min}$, sensitivity: 40, red cell level (RCL): 40, pressplate force: $8 \mathrm{~kg}$.

The contents of 10 ABO-identical CPD buffy coats were mixed and split equally into two $600-\mathrm{mL}$ pooling containers. A volume of $\approx 300 \mathrm{~mL}$ of acetate containing additive solution (PAS II) was added to each pool (containing the equivalent of five buffy coats). The pools were sampled for cell counts and haematocrit measures, connected to a platelet storage container and centrifuged $(655 \mathrm{~g})$ for $5 \mathrm{~min}$. From each pair of pools one platelet concentrate was prepared using the Optipress II as extractor while the other pool was processed with a manual method using a conventional plasma extractor. Sealing was in both cases performed with a hand-held sealer. Each platelet concentrate has been assessed for volume, all cell counts and free haemoglobin at the time of separation.

\section{Determination of the maximum pressplate force setting}

Primary separation was performed with machine settings defined as mentioned above except for the press plate force. The pressplate force was adjusted to the $50-\mathrm{kg}$ setting instead of the $25-\mathrm{kg}$ setting evaluated in the primary separation study in order to investigate whether the speed of blood processing could be increased. The

(C) 1998 Blackwell Science Ltd, Transfusion Medicine, 8, 319-324 
Table 1. Results of the primary separation study with the Optipress ${ }^{\circledR}$ II

\begin{tabular}{|c|c|c|c|c|c|}
\hline & & \multirow[b]{2}{*}{ Units } & \multicolumn{3}{|c|}{ Optipress ${ }^{\circledR}$ II } \\
\hline & & & $n$ & Mean & SD \\
\hline \multirow[t]{4}{*}{ Whole blood } & volume & $\mathrm{mL}$ & 31 & $561 \cdot 9$ & 8 \\
\hline & leukocytes & $10^{9}$ per unit & 31 & $3 \cdot 34$ & $0 \cdot 88$ \\
\hline & platelets & $10^{9}$ per unit & 31 & 122 & 26 \\
\hline & erythrocytes & $10^{12}$ per unit & 31 & $2 \cdot 35$ & $0 \cdot 19$ \\
\hline \multirow[t]{6}{*}{ Red cell concentrate } & volume & $\mathrm{mL}$ & 31 & $311 \cdot 5$ & $13 \cdot 3$ \\
\hline & leukocytes & $10^{9}$ per unit & 31 & 0.59 & $0 \cdot 46$ \\
\hline & platelets & $10^{9}$ per unit & 31 & $1 \cdot 35$ & $0 \cdot 37$ \\
\hline & erythrocytes & $10^{12}$ per unit & 31 & $2 \cdot 04$ & $0 \cdot 18$ \\
\hline & haematocrit & $\%$ & 31 & 61 & 2 \\
\hline & free $\mathrm{Hb}$ (day 35 ) & mg per unit & 31 & $77 \cdot 05$ & $26 \cdot 96$ \\
\hline \multirow[t]{4}{*}{ Plasma } & volume & $\mathrm{mL}$ & 31 & $307 \cdot 8$ & $14 \cdot 58$ \\
\hline & leukocytes & $10^{6}$ per unit & 31 & $1 \cdot 19$ & $1 \cdot 17$ \\
\hline & platelets & $10^{9}$ per unit & 31 & $3 \cdot 36$ & $1 \cdot 01$ \\
\hline & free $\mathrm{Hb}$ & mg per unit & 31 & $4 \cdot 78$ & $3 \cdot 85$ \\
\hline \multirow[t]{5}{*}{ Buffy coats } & volume & $\mathrm{mL}$ & 31 & $54 \cdot 4$ & $1 \cdot 7$ \\
\hline & leukocytes & $10^{9}$ per unit & 31 & $2 \cdot 77$ & 0.58 \\
\hline & platelets & $10^{9}$ per unit & 31 & $91 \cdot 7$ & $18 \cdot 3$ \\
\hline & erythrocytes & $10^{12}$ per unit & 31 & $0 \cdot 29$ & $0 \cdot 02$ \\
\hline & haematocrit & $\%$ & 31 & $51 \cdot 2$ & $3 \cdot 7$ \\
\hline
\end{tabular}

sampling was performed as mentioned above in the primary separation study.

\section{Optipress II efficiency study}

Upon definition of the force parameter setting mentioned for the primary separation study, Optipress II machines were set with $25 \mathrm{~kg}$ pressplate force.

The machines were then used on a routine basis for 6 weeks to allow an estimation of machine efficiency and its impact on product quality.

Comparison has been established with the Optipress I equipment on the following aspects: duration of the separation process, total time for the centrifugation, the separation and the tube sealing of a quantity of blood packs corresponding to eight centrifuge machine loads (96 units) and the scrap levels of the separation process.

\section{Comparison with Optipress I during routine production}

The Optipress II was then used in production with parameter settings defined during the primary separation study.

Routine quality control results of the blood components produced with the Optipress II during quarters 3 and 4 in 1997 were compared with the same data generated during the routine usage of the Optipress I during quarters 3 and 4 in 1995.

\footnotetext{
(C) 1998 Blackwell Science Ltd, Transfusion Medicine, 8, 319-324
}

\section{Data treatment}

For the primary separation study the data of routine production on Optipress II were compared to those obtained from quality control testing during the last two quarters of 1995 using a $t$-test (difference between two means Statistica for Windows, version 4.5).

For the secondary separation study platelet characteristics were evaluated using a $t$-test (paired two samples for means Microsoft Excel, version 5·0). The Optipress II efficiency study results were analysed using a $t$-test (two samples for means Microsoft Excel, version 5·0). $P$ values for the comparison of routine results were calculated using a significance test difference between two means (Statistica for Windows version 5.1 StatSoft Inc.).

\section{RESULTS}

\section{Primary separation study}

The results of the primary separation study with Optipress II are shown in Table 1. Comparison of the quality control results in routine production of Optipress I and of Optipress II is shown in Table 2.

Altough routine quality control data do not include cell counts on donation and consequently do not allow us to draw definite conclusions on the trial results, the 
Table 2. Comparison of the quality control results in routine production of the Optipress ${ }^{\circledR}$ I and of the Optipress ${ }^{\circledR}$ II

\begin{tabular}{|c|c|c|c|c|c|c|c|c|c|}
\hline & & \multirow[b]{2}{*}{ Units } & \multicolumn{3}{|c|}{ Optipress ${ }^{\circledR} \mathrm{I}$} & \multicolumn{4}{|c|}{ Optipress ${ }^{\circledR}$ II } \\
\hline & & & $n$ & Mean & SD & $n$ & Mean & SD & $P$ \\
\hline \multirow[t]{4}{*}{ Whole blood } & volume & $\mathrm{mL}$ & 42780 & 574 & 10 & $43 \cdot 784$ & 568 & 10 & $<0.0001$ \\
\hline & leukocytes & $10^{9}$ per unit & & & & & & & \\
\hline & platelets & $10^{9}$ per unit & & & & & & & \\
\hline & erythrocytes & $10^{12}$ per unit & & & & & & & \\
\hline \multirow[t]{6}{*}{ Red cell concentrate } & volume & $\mathrm{mL}$ & 474 & 307 & 17 & 520 & 302 & 16 & $<0.0001$ \\
\hline & leukocytes & $10^{9}$ per unit & 474 & $0 \cdot 52$ & $0 \cdot 36$ & 520 & $0 \cdot 64$ & $0 \cdot 39$ & $<0.0001$ \\
\hline & platelets & $10^{9}$ per unit & 474 & $3 \cdot 2$ & $0 \cdot 8$ & 520 & $0 \cdot 7$ & 0.7 & $<0.0001$ \\
\hline & erythrocytes & $10^{12}$ per unit & 474 & 2 & $0 \cdot 19$ & 520 & 1.99 & $0 \cdot 19$ & NS \\
\hline & haematocrit & $\%$ & 474 & 60 & 3 & 520 & 59 & 2 & $<0.0001$ \\
\hline & free $\mathrm{Hb}$ (day 35) & mg per unit & 120 & $67 \cdot 8$ & $36 \cdot 3$ & 135 & 21 & 16 & $<0.0001$ \\
\hline \multirow[t]{4}{*}{ Plasma } & volume & $\mathrm{mL}$ & 474 & 319 & 17 & 520 & 314 & 17 & $<0.0001$ \\
\hline & leukocytes & $10^{6}$ per unit & 120 & $2 \cdot 6$ & 3 & 135 & $2 \cdot 6$ & $2 \cdot 7$ & NS \\
\hline & platelets & $10^{9}$ per unit & 475 & 5 & $2 \cdot 6$ & 520 & 3 & $1 \cdot 7$ & $<0.0001$ \\
\hline & free $\mathrm{Hb}$ & mg per unit & 475 & $4 \cdot 7$ & $5 \cdot 1$ & 519 & $5 \cdot 2$ & 4.9 & NS \\
\hline \multirow[t]{5}{*}{ Buffy coats } & volume & $\mathrm{mL}$ & 490 & 54 & 4 & 515 & 55 & 2 & $<0.0001$ \\
\hline & leukocytes & $10^{9}$ per unit & 490 & $3 \cdot 03$ & 0.73 & 515 & $2 \cdot 28$ & 0.64 & $<0.0001$ \\
\hline & platelets & $10^{9}$ per unit & 490 & 96 & 20 & 515 & 106 & 25 & $<0.0001$ \\
\hline & erythrocytes & $10^{12}$ per unit & 490 & $0 \cdot 29$ & 0.04 & 515 & $0 \cdot 30$ & $0 \cdot 03$ & $<0.0001$ \\
\hline & haematocrit & $\%$ & 420 & 50 & 4 & 515 & 49 & 3 & $<0.0001$ \\
\hline
\end{tabular}

following appears from the data analysis:

- The platelet content of red cell concentrates $\left(0.7 \pm 0.7 \times 10^{9}\right.$ per unit) produced with the new extractor in routine practice is significantly $(P<0.0001)$ lower than the values with Optipress I $\left(3 \cdot 20 \pm 0.08 \times 10^{9}\right.$ per unit).

- Haemolysis in the red cell concentrates was assessed by measure of free haemoglobin and was found to be within acceptable limits with respective average values of $0.05 \%(n=31$ at day 1$), 0 \cdot 14 \%(n=31$ at day 14$)$ and $0.22 \%(n=10$ at day 35$)$.

- The platelet content of the plasma fractions produced routinely with the Optipress II appears significantly lower than that seen with the Optipress I $\left(5 \pm 2.6 \times 10^{9}\right.$ platelets per unit $P<0.0001)$.

Finally, the new separator has a better reproducibility $(P<0.005-$ Fisher test $)$ in buffy coat volume $(55 \pm 2 \mathrm{~mL}$ compared to $54.0 \pm 4.0 \mathrm{~mL}$ ) than the actual model in use in the bloodbank laboratory.

\section{Platelet concentrate preparation study}

Statistical analysis shows equivalence of data obtained on platelet concentrates separated with the Optipress II compared to platelet concentrates prepared using the manual method from the bloodbank laboratory.
Quality of blood components prepared with pressplate force at maximum setting of $50 \mathrm{~kg}$

Comparing results on blood quality components produced with the maximum pressplate force $(50 \mathrm{~kg}$ setting value) with similar data obtained from the first part of the Optipress II study revealed a greater variation in buffy coat volume, significantly higher platelet content and haemolysis in the red cell concentrates (data not shown).

\section{Duration of the separation process}

Mean separation time for the Optipress II is $340 \mathrm{~s}$ compared to $324 \mathrm{~s}$ for the similar operation on the Optipress I, but statistical analysis shows no significant difference either in the distribution or in the mean value.

\section{Total time for the separation processing}

The total time to process eight centrifuge loads using the Optipress II was compared to the time needed for the same operation using the Optipress I. This comparison ( $1 \mathrm{~h}, 24 \mathrm{~min}$ and $10 \mathrm{~s}$ for the Optipress II and $1 \mathrm{~h} 24 \mathrm{~min}$ and $36 \mathrm{~s}$ for the Optipress I) does not reveal a difference between the two machines.

(C) 1998 Blackwell Science Ltd, Transfusion Medicine, 8, 319-324 
Table 3. Characteristics of five donor unit platelet concentrates produced by a blood processor in comparison with the manual method $(n=12)$

\begin{tabular}{|c|c|c|c|c|c|}
\hline & $\begin{array}{l}\text { Plt conc. } \\
\text { volume }\end{array}$ & $\begin{array}{l}\text { Plt conc. } \\
\text { RBC }\end{array}$ & $\begin{array}{l}\text { Plt conc. } \\
\text { WBC }\end{array}$ & $\begin{array}{l}\text { Plt conc. } \\
\text { Plt }\end{array}$ & \multirow{2}{*}{$\begin{array}{l}\text { Plt conc. } \\
\text { free } \mathrm{Hb} \\
\text { day } 1 \\
\left(\mathrm{mg} \mathrm{L}^{-1}\right)\end{array}$} \\
\hline & $(\mathrm{mL})$ & ( $10^{12}$ per unit) & ( $10^{9}$ per unit) & $\left(10^{9}\right.$ per unit $)$ & \\
\hline \multicolumn{6}{|c|}{ Optipress ${ }^{\circledR}$ II } \\
\hline Mean & $352 \cdot 9$ & $0 \cdot 01$ & 0.09 & $368 \cdot 6$ & $101 \cdot 0$ \\
\hline SD & $6 \cdot 4$ & $0 \cdot 00$ & 0.03 & $29 \cdot 5$ & $64 \cdot 9$ \\
\hline Max. & $367 \cdot 2$ & $0 \cdot 01$ & $0 \cdot 13$ & $419 \cdot 2$ & $280 \cdot 0$ \\
\hline Min. & 343.4 & $0 \cdot 00$ & $0 \cdot 06$ & $319 \cdot 7$ & $48 \cdot 6$ \\
\hline \multicolumn{6}{|c|}{ Manual method } \\
\hline Mean & $353 \cdot 4$ & $0 \cdot 01$ & 0.09 & $368 \cdot 6$ & $128 \cdot 8$ \\
\hline SD & $6 \cdot 5$ & $0 \cdot 00$ & 0.03 & $30 \cdot 8$ & $84 \cdot 6$ \\
\hline Max. & $366 \cdot 2$ & $0 \cdot 01$ & $0 \cdot 13$ & $414 \cdot 2$ & $370 \cdot 0$ \\
\hline Min. & $343 \cdot 4$ & $0 \cdot 00$ & $0 \cdot 05$ & $314 \cdot 2$ & $57 \cdot 4$ \\
\hline
\end{tabular}

\section{Scrap level comparison}

A comparison of defect rates was made over a period of 6 weeks during which the Optipress II was used on a routine basis against data gathered in 1996 during use of Optipress I and manual tubes sealing for an equivalent period of time (see Table 4).

Two parameters were used for the comparison:

1 incidence of leaking seals;

2 incidence of incorrect position of the buffy coat interface level at the end of the separation process resulting in inappropriate buffy coat composition (haematocrit, white blood cells, red blood cells and platelet content).

The results of the comparison are shown in Table 3.

\section{DISCUSSION}

The application of good manufacturing practices requires standardized methods of production. The use of automated blood processing techniques reduces the impact of human factors in blood component preparation and improves standardization and quality of blood products.

The primary separation process using bottom and top

Table 4. Scrap level comparison between the two automated blood processors

\begin{tabular}{llrr}
\hline & $\begin{array}{l}\text { Quantity of } \\
\text { donations }\end{array}$ & Leaking seals & $\begin{array}{r}\text { Incorrect } \\
\text { BC level }\end{array}$ \\
\hline 1996 Optipress $^{\circledR}$ I & 9281 & $41(0.44 \%)$ & $178(1.92 \%)$ \\
Optipress $^{\circledR}$ II & 9432 & $0(0.00 \%)$ & $12(0.31 \%)$ \\
\hline
\end{tabular}

(c) 1998 Blackwell Science Ltd, Transfusion Medicine, 8, 319-324 containers allowed the production of blood components with characteristics within tight limits. Using the second generation blood processor, red cell concentrates were produced with a mean leucocyte reduction of $82 \%$, a platelet reduction of $99 \%$ and a red cell loss of only $13 \%$ compared with whole blood (Heaton et al., 1997). The platelet concentrates produced from buffy coats contained $63 \%$ of the original whole blood platelet content and only $3 \%$ of residual leucocytes (without filtration).

Together with the very low leucocyte and platelet content of the plasma, a very efficient component separation is achieved in this way and compares favourably with the existing literature (Meryman \& Hornblower, 1986; Loos, 1988; Högman et al., 1992).

The same second generation blood processor that is used for primary separation of blood components can be used for the secondary separation of platelet concentrates from pooled buffy coats, further reducing the influence of the human factor on the variability of product characteristics in routine large-scale platelet concentrate production (Pietersz et al., 1990; Rebulla et al., 1990; American Association of Blood Banks, 1994; Council of Europe, 1995; Zoon et al., 1996).

The results obtained demonstrate the advantage of a full separation process of automation with a drastic reduction of tube sealing defects. The improvement in the optical detection system also has a positive effect on the consistency of the buffy coat contents.

Further improvements in machine efficiency can still be made. However, the quality of blood components prepared with pressplate force at maximum setting of $50 \mathrm{~kg}$ did not result in optimal products. Further optimization of the operating parameters and some adaptations 
of the disposable design may be necessary to operate the machine at maximum force without inducing higher haemolysis. On the other hand, the results of the comparison on time needed to handle several centrifuge loads through the separation process may indicate that the machine processing time is not the limiting factor in the separation laboratory efficiency.

In conclusion, improvement in standardization of blood component preparation is possible with an automated blood processor, leading to improvement in the quality of blood products for patient care.

\section{REFERENCES}

American Association of Blood Banks. (1994) 16th Edition Standards for Blood Banks and Transfusion Services. Bethesda, MD

Council of Europe. (1995) Guide to the Preparation, Use and Quality Assurance of Blood Components, Strasbourg. Council of Europe Press.

Gulliksson, H., Eriksson, L., Högman, C.F., et al. (1995) Buffy-coat-derived platelet concentrates prepared from half-strength citrate CPD and CPD whole-blood units. Comparison between three additive solutions: in vitro studies. Vox Sanguinis, 68, 152-159.

Heaton, W.A.L., Rebulla, P., Pappalettera, M. \& Dzik, W.H.A. (1997) Comparative analysis of different methods for routine blood component preparation. Transfusion Medicine Reviews, 11, 116-129.

Högman, C.F., Eriksson, L. \& Ring, M. (1992) Automated blood component preparation with the Opti-system: three years experience. Transfusionsmedizin: Beitr Infusionsther, 30, 100-107.
Högman, C.F., Hedlund, K. \& Zetterstrom, H. (1978) Clinical usefulness of red cells preserved in protein-poor mediums. New England Journal of Medicine, 299, 1377-1382.

Loos, J.A. (1988) Standardization procedures required by automation of blood component preparation. In: Automation in Blood Transfusion. Proceedings of the Thirteenth International Symposium on Blood Transfusion. Kluwer, Dodrecht, the Netherlands: 105-107.

Lovric, V.A., Bryant, J. \& Parker, A. (1977) Improved quality of packed cells. Medical Journal of Australia, 2, 183-186.

Meryman, H.T. \& Hornblower, M. (1986) The preparation of red cells depleted of leukocytes. Transfusion, 26, 101-106.

Molijn, M.H.J., van Rhenen, D.J., van Toledo, P.J. \& Vermeij, J. (1994) An increase in blood component preparation by 15000 a year without staff expansion by introduction of Optipac $^{\circledR} /$ Optipress ${ }^{\circledR}$. Vox Sanguinis, 67 (S2), 94, no. 0163.

Pietersz, R.N.I., Prins, H.K., Dekker, W.J.A. et al. (1990) Leukocyte-poor platelet concentrates from buffy coats. The Dutch Experience. Transfusion Science, 11, 349-351.

Rebulla, P., Bertolini, P., Riccardia, D., et al. (1990) Platelet concentrates prepared from pooled buffy coats and stored in a glucose-free crystalloid medium. The Milan Experience. Transfusion Science, 11, 357-362.

van Rhenen, D.J., Vermeij, J., Kappers-Klunne, M.C. \& Payrat, J.M. (1995) Evaluation of a new citrate-acetate- $\mathrm{NaCl}$ platelet additive solution for the storage of white cell reduced platelet concentrates obtained from half strength CPD pooled buffy coats. Transfusion, 35, 50-53.

Zoon, K.C. (ed.) (1996) Recommendations and licensure requirements for leukocyte-reduced blood products. Memorandum from director, Center of Biologics Evaluation and Research Department of Health, Human Services, United States Food and Drug Administration. Washington, DC. 